\title{
Simplifying the use of prognostic information in traumatic brain injury. Part 2: Graphical presentation of probabilities
}

\author{
Gordon D. Murray, MA, PhD, ${ }^{1}$ Paul M. Brennan, MBBChir, FRCS, $\mathrm{PhD},{ }^{2}$ and \\ Graham M. Teasdale, MBBS, FRCP, FRCS ${ }^{3}$ \\ ${ }^{1}$ Usher Institute of Population Health Sciences and Informatics and ${ }^{2}$ Centre for Clinical Brain Sciences, University of Edinburgh; \\ and ${ }^{3}$ Institute of Health and Wellbeing, University of Glasgow, United Kingdom
}

OBJECTIVE Clinical features such as those included in the Glasgow Coma Scale (GCS) score, pupil reactivity, and patient age, as well as CT findings, have clear established relationships with patient outcomes due to neurotrauma. Nevertheless, predictions made from combining these features in probabilistic models have not found a role in clinical practice. In this study, the authors aimed to develop a method of displaying probabilities graphically that would be simple and easy to use, thus improving the usefulness of prognostic information in neurotrauma. This work builds on a companion paper describing the GCS-Pupils score (GCS-P) as a tool for assessing the clinical severity of neurotrauma.

METHODS Information about early GCS score, pupil response, patient age, CT findings, late outcome according to the Glasgow Outcome Scale, and mortality were obtained at the individual adult patient level from the CRASH (Corticosteroid Randomisation After Significant Head Injury; $n=9045$ ) and IMPACT (International Mission for Prognosis and Clinical Trials in TBI; $n=6855$ ) databases. These data were combined into a pooled data set for the main analysis. Logistic regression was first used to model the combined association between the GCS-P and patient age and outcome, following which CT findings were added to the models. The proportion of variability in outcomes "explained" by each model was assessed using Nagelkerke's R².

RESULTS The authors observed that patient age and GCS-P have an additive effect on outcome. The probability of mortality 6 months after neurotrauma is greater with increasing age, and for all age groups the probability of death is greater with decreasing GCS-P. Conversely, the probability of favorable recovery becomes lower with increasing age and lessens with decreasing GCS-P. The effect of combining the GCS-P with patient age was substantially more informative than the GCS-P, age, GCS score, or pupil reactivity alone. Two-dimensional charts were produced displaying outcome probabilities, as percentages, for 5 -year increments in age between 15 and 85 years, and for GCS-Ps ranging from 1 to 15; it is readily seen that the movement toward combinations at the top right of the charts reflects a decreasing likelihood of mortality and an increasing likelihood of favorable outcome.

Analysis of CT findings showed that differences in outcome are very similar between patients with or without a hematoma, absent cisterns, or subarachnoid hemorrhage. Taken in combination, there is a gradation in risk that aligns with increasing numbers of any of these abnormalities. This information provides added value over age and GCS-P alone, supporting a simple extension of the earlier prognostic charts by stratifying the original charts in the following $3 \mathrm{CT}$ groupings: none, only 1 , and 2 or more CT abnormalities.

CONCLUSIONS The important prognostic features in neurotrauma can be brought together to display graphically their combined effects on risks of death or on prospects for independent recovery. This approach can support decision making and improve communication of risk among health care professionals, patients, and their relatives. These charts will not replace clinical judgment, but they will reduce the risk of influences from biases.

https://thejns.org/doi/abs/10.3171/2017.12.JNS172782

KEYWORDS Glasgow Coma Scale; head injury; traumatic brain injury; trauma; prognosis; charts

ABBREVIATIONS CRASH = Corticosteroid Randomisation After Significant Head Injury; GCS = Glasgow Coma Scale; GCS-P = GCS-Pupils score; GCS-PA = GCS-P plus age; GCS-PA CT = GCS-P plus age plus CT findings; GOS = Glasgow Outcome Scale; IMPACT = International Mission for Prognosis and Clinical Trials in Traumatic Brain Injury; PRS = pupil reactivity score; $\mathrm{SAH}=$ subarachnoid hemorrhage; $\mathrm{TBI}$ = traumatic brain injury.

SUBMITTED November 4, 2017. ACCEPTED December 23, 2017.

INCLUDE WHEN CITING Published online April 10, 2018; DOI: 10.3171/2017.12.JNS172782. 


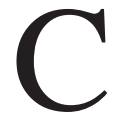
LINICIANS' decisions about the management of acute head injury are influenced by their perceptions of the patient's prognosis. ${ }^{1}$ Nevertheless, despite several decades of interest in, and descriptions of, more than 100 methods of making probabilistic predictions of an individual patient's outcome, ${ }^{39}$ there is little evidence that these methods have found a role in clinical practice in neurotrauma. ${ }^{22,35,39}$ This position is echoed in other acute illnesses, ${ }^{48}$ for example, predicting outcomes in acute stroke ${ }^{12}$ or pulmonary embolism, ${ }^{3}$ or predicting hospital readmission. ${ }^{6,19}$ A survey of neurosurgical units in the United Kingdom revealed that almost all clinicians (90\%) use the Glasgow Coma Scale as an index of injury severity on referral or hospital admission; in no instances were further formal multifactorial calculations of prognosis being made.

A reason for the lack of uptake of probabilistic models may be that clinicians can be uneasy about dealing with explicit mathematical probabilities. This is especially the case if the process of producing models with varying, usually unquantified, uncertainty seems opaque and lacks a context for the information. However, whatever the reasons limiting uptake of probabilistic models, clinical care itself is exposed to the influence of personal, highly variable subjective opinions, ${ }^{32}$ and more effective and acceptable methods of communicating prognoses are required in patients' clinical care.

Aids to the understanding of risks through graphical presentation have been found useful in presenting information in several fields, improving diagnostic inferences and judgment. ${ }^{5,14}$ Graphical aids may provide a simpler assessment of risk than more complicated models, but they trade off this loss of detail for greater transparency and an understanding of the underlying pattern of relationships between features, both singly and in combination, and the outcome of interest.

A visual aid may improve the usefulness of probabilistic models in neurotrauma. Four prognostic factors contain much of the information needed for prognosis of patients with acute head injury. 2,733 The age of the patient is a powerful factor. Most clinical predictive information is contained in the patient's score on the Glasgow Coma Scale and pupil reaction; and CT findings are the most useful investigative index. These factors are key features in most predictive systems..$^{22,35,49}$ We therefore investigated ways in which these 4 factors could be combined to convey information about the risks of mortality, or, conversely, the prospects for independent recovery, after head injury. Our intent was to create a simple graphical display that would be easy to use in a clinical setting.

\section{Methods}

\section{Sources of Information}

Our investigations were based on information derived from 2 large head-injury databases. The CRASH (Corticosteroid Randomisation After Significant Head Injury) study recruited 10,008 adults with head injury and GCS scores of 14 or less from 239 hospitals in 49 countries. $^{43}$ The IMPACT (International Mission for Prognosis and Clinical Trials in Traumatic Brain Injury) prognostic model was developed using data from 11,989 patients who had sustained moderate (GCS scores 9-12) or severe (GCS scores 3-8) traumatic brain injuries (TBIs): the data had been collected prospectively during 11 different studies. ${ }^{28}$ The present study used pooled data derived from a combined database, which was constructed as described in our companion paper. ${ }^{4}$

\section{Prognostic Features}

We abstracted items from the CRASH and IMPACT databases into a single data set containing information on patient age in years at the time of injury and clinical findings.

\section{Patient Age}

We restricted our analysis to patients 16 years of age or older. Age was treated as a linear factor on a logistic scale, as it was previously demonstrated that the association between age and both mortality and unfavorable outcome in neurotrauma can be well described in this way. ${ }^{17}$

\section{GCS Score}

In the CRASH study the eye, verbal, and motor components of the Glasgow Coma Scale were recorded for each patient at the time of randomization, from which the GCS total score was calculated. In each of the 11 studies included in the IMPACT database the GCS score was determined at different time periods and the IMPACT model was developed using a "derived GCS score," defined as the GCS score obtained closest to randomization or on entry into the study. ${ }^{28}$

\section{Pupil Reactivity}

Pupil reaction to light was coded separately for each eye and was recorded at the same time as assessment of the GCS score. To combine the GCS score and pupil findings, a GCS-Pupils score (GCS-P) was derived, as described in the companion paper, ${ }^{4}$ by subtracting a pupil reactivity score (PRS) from the GCS total score (range 3-15): GCS-P = GCS score - PRS. The PRS is determined from the pupillary reaction to light. If both pupils are reactive, the PRS equals zero. If 1 pupil is unreactive the PRS equals 1. If both pupils are unreactive the PRS equals 2 . This gives a possible range of GCS-P values from 1 to 15 .

\section{CT Scan}

The methods used to record findings of the patient's first CT scan after arrival at the hospital differed between the CRASH and IMPACT databases. In the CRASH study, the presence or absence of normal appearances, petechial hemorrhage, obliteration of the third ventricle and/or basal cisterns, subarachnoid hemorrhage (SAH), midline shift greater than $5 \mathrm{~mm}$, or any type of intracranial hematoma (evacuated or not) were recorded as separate items ${ }^{43}$ In the IMPACT database the findings were recorded according to the Marshall classification as follows: I) diffuse injury, no visible pathology; II) diffuse injury, cisterns present, midline shift 0-5 mm; III) swelling, cisterns compressed or absent; IV) diffuse injury and midline shift $>5 \mathrm{~mm}$; [V]) evacuated mass lesion; or [VI]) nonevacuated mass 
lesion. ${ }^{21}$ The presence or absence of SAH was also recorded.

To take account of these differences in recording, and in accord with their prognostic value, CT data were abstracted from the CRASH and IMPACT databases and categorized according to the presence or absence of 3 abnormalities: 1) an intracranial hematoma (evacuated or not), 2) absent cisterns, and 3) SAH. ${ }^{41,51}$

\section{Patient Outcome}

In the CRASH study, outcome on the Glasgow Outcome Scale (GOS) was determined at 6 months postinjury by using a structured postal questionnaire. For data in the IMPACT database, outcome on the GOS was derived for each patient 6 months after head injury based on the available information. If 6-month data were unavailable, the 3 -month GOS classification was used. Data were extracted from the 6-month (3-month if necessary) GOS findings, and "favorable outcome" was defined as moderate disability or good recovery on the GOS. We included the 140 patients listed as being in a persistent vegetative state $(1.3 \%)$ in the mortality group.

\section{Analysis and Modeling of Relationships}

The modeling that led to the combination of the GCS total score and pupil reactivity into the GCS-P is set out in our companion paper. ${ }^{4}$ Logistic regression was used first to model the combined association between the GCS-P and patient age and outcome; following this, CT findings were added to the models. An assessment of the information yield from combinations of the prognostic features was made. The proportion of variability in outcome that were "explained" by each model was assessed using Nagelkerke's R ${ }^{2} .36$

Once a model was selected, we constructed a prognostic chart using predicted probabilities of either mortality or favorable outcome, which were derived from the fitted model. We then constructed a table providing these predicted probabilities over a grid corresponding to each possible GCS-P value and 5-year steps in age from 15 to 85 years. Color shading was used to highlight gradations in the relevant predicted probabilities from $0 \%$ up to $100 \%$.

The performance of predictions presented in these charts was compared with the performance of the published IMPACT ${ }^{49}$ and CRASH $^{38}$ models (the IMPACT Core and Extended models and the CRASH Basic and CT models) by calculating the corresponding $\mathrm{R}^{2}$ values for patients in our database, whose information came separately from the IMPACT and CRASH databases. Data were not available to cross-apply IMPACT models to CRASH patients or cross-apply CRASH models to IMPACT patients.

\section{Results}

\section{Patient Review}

We identified patients in each database for whom information on age, GCS score, pupil reactivity, and CT findings was all available. We excluded patients under 16 years of age $(n=334)$. For our analysis, the information from the 2 sources was combined into a single set of 10,702 patients with features shown in Table 1.
TABLE 1. Principal patient characteristics for the analysis data set

\begin{tabular}{lc}
\hline \multicolumn{1}{c}{ Factor } & Value \\
\hline No. of patients & 10,702 \\
\hline Mean age in yrs (SD) & $36.6(16.5)$ \\
\hline M/F ratio & $80: 20$ \\
\hline GCS score & $1796(16.8)$ \\
\hline $13-15$ (mild head injury) & $2653(24.8)$ \\
\hline 9-12 (moderate head injury) & $6253(58.4)$ \\
\hline $3-8$ (severe head injury) & $1169(10.9)$ \\
\hline No. of reactive pupils & $1026(9.6)$ \\
\hline 0 & $8507(79.5)$ \\
\hline 1 & \\
\hline 2 & $1817(17.0)$ \\
\hline CT classification & $3997(37.3)$ \\
\hline Normal & $4888(45.7)$ \\
\hline Hematoma & $2678(25.0)$ \\
\hline Other & $6257(58.5)$ \\
\hline Mortality &
\end{tabular}

Unless otherwise indicated, values represent number of patients (\%). Mortality group includes patients listed as being in a persistent vegetative state.

\section{Relationship of Individual Prognostic Features to Patient Outcome}

Patient Age

The relationship between increasing age above 16 years and mortality or favorable outcome is set out in Table 2 and illustrated in Fig. 1. The probability of death 6 months after head injury increases steadily, and the probability of favorable recovery decreases steadily, as age above 16 years increases.

TABLE 2. Patient age and outcomes

\begin{tabular}{cccc}
\hline Age $(\mathrm{yrs})$ & Sample Size & Mortality & Favorable Outcome \\
\hline $16-20$ & 1868 & $342(18.3)$ & $1269(67.9)$ \\
\hline $21-25$ & 1775 & $324(18.3)$ & $1211(68.2)$ \\
\hline $26-30$ & 1360 & $260(19.1)$ & $898(66.0)$ \\
\hline $31-35$ & 1059 & $232(21.9)$ & $659(62.2)$ \\
\hline $36-40$ & 977 & $217(22.2)$ & $601(61.5)$ \\
\hline $41-45$ & 821 & $207(25.2)$ & $440(53.6)$ \\
\hline $46-50$ & 742 & $231(31.1)$ & $359(48.4)$ \\
\hline $51-55$ & 560 & $160(28.6)$ & $278(49.6)$ \\
\hline $56-60$ & 494 & $165(33.4)$ & $215(43.5)$ \\
\hline $61-65$ & 347 & $127(36.6)$ & $141(40.6)$ \\
\hline $66-70$ & 214 & $105(49.1)$ & $77(36.0)$ \\
\hline $71-75$ & 211 & $115(54.5)$ & $61(28.9)$ \\
\hline $76-80$ & 137 & $94(68.6)$ & $31(22.6)$ \\
\hline $81-85$ & 77 & $53(68.8)$ & $11(14.3)$ \\
\hline $86+$ & 60 & $46(76.7)$ & $6(10.0)$ \\
\hline
\end{tabular}

Unless otherwise indicated, values represent number of patients (\%). 


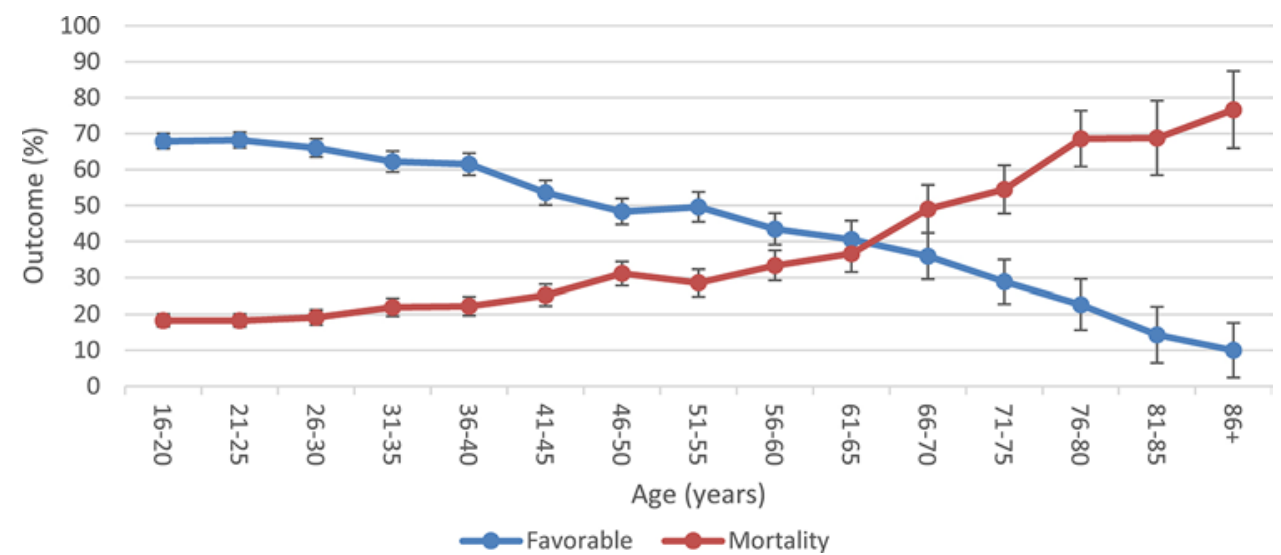

FIG. 1. Percentages of patients dead (Mortality) or with a favorable outcome (Favorable) 6 months following head injury according to patient age at hospital admission. Error bars show corresponding $95 \%$ confidence intervals (Cls). Figure is available in color online only.

\section{GCS Score and Pupil Reactivity}

We combined data on the GCS score and pupil reactivity by using the GCS-P. The interaction between the GCS-P and either mortality or favorable outcome is set out in Table 3. The probability of death 6 months after head injury increases and the probability of favorable recovery decreases as the GCS-P declines from 15 to 1 . This is illustrated in Fig. 2.

\section{Interaction Between Age, GCS-P Score, and Outcome}

We observed that patient age and the GCS-P have an additive effect on outcome. For GCS-P values from 1 to 15 , the probability of death 6 months after head injury becomes greater with increasing age, and for all age groups the probability of death is greater with decreasing GCS-P. Conversely, the probability of favorable recovery decreases with increasing age and decreases with lower-

TABLE 3. GCS-P and outcome

\begin{tabular}{cccc}
\hline GCS-P & Sample Size & Mortality & Favorable Outcome \\
\hline 1 & 298 & $235(78.9)$ & $32(10.7)$ \\
\hline 2 & 384 & $270(70.3)$ & $52(13.5)$ \\
\hline 3 & 719 & $329(45.8)$ & $215(29.9)$ \\
\hline 4 & 709 & $283(39.9)$ & $217(30.6)$ \\
\hline 5 & 821 & $279(34.0)$ & $332(40.4)$ \\
\hline 6 & 1139 & $297(26.1)$ & $596(52.3)$ \\
\hline 7 & 1443 & $298(20.7)$ & $879(60.9)$ \\
\hline 8 & 813 & $165(20.3)$ & $532(65.4)$ \\
\hline 9 & 619 & $114(18.4)$ & $431(69.6)$ \\
\hline 10 & 671 & $116(17.3)$ & $481(71.7)$ \\
\hline 11 & 616 & $81(13.1)$ & $467(75.8)$ \\
\hline 12 & 700 & $80(11.4)$ & $541(77.3)$ \\
\hline 13 & 823 & $76(9.2)$ & $672(81.7)$ \\
\hline 14 & 912 & $50(5.5)$ & $782(85.7)$ \\
\hline 15 & 35 & $5(14.3)$ & $28(80.0)$ \\
\hline
\end{tabular}

Unless otherwise indicated, values represent number of patients (\%). ing GCS-P score. This is shown in Tables 4 and 5. In patients 16-20 years of age with a GCS-P of 15 , the rate of mortality was $0 \%$ and the rate of favorable recovery was $100 \%$. In patients older than 85 years with a GCS-P of 1, the rate of mortality was $100 \%$ and the rate of favorable recovery was $0 \%$, although the numbers of patients with these extreme combinations were very small. There was no evidence of a threshold or break point at which there was a change in the relationship.

There are potentially $225(15 \times 15)$ possible combinations of age (in 5-year increments) and GCS-P; so despite the size of the data set, the numbers in some combinations are limited. We therefore used logistic regression to attempt to illustrate the underlying smooth relationship between patient age, GCS-P, and outcome. The resulting fitted models, in which both age and GCS-P were taken as continuous variables, are demonstrated graphically in Fig. 3 left for mortality and in Fig. 3 right for favorable recovery.

Nagelkerke's $\mathrm{R}^{2}$ is a measure of the proportion of variability in outcome that is explained by the model used to

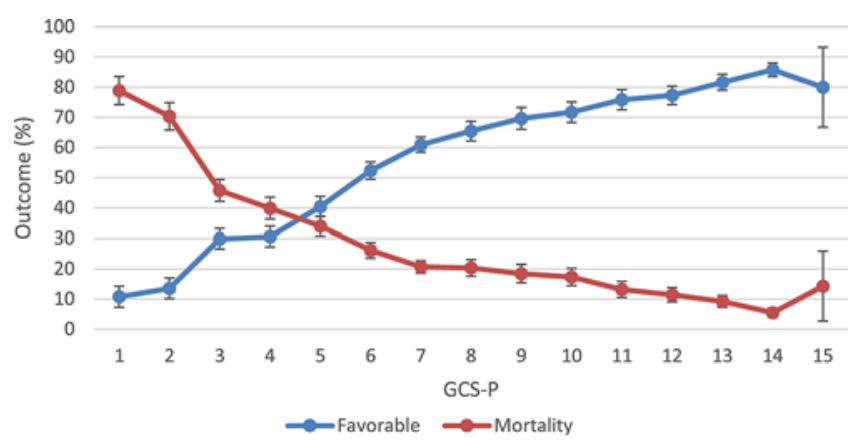

FIG. 2. Relationship between the GCS-P and the percentages of patients dead or with favorable recovery 6 months after injury. Error bars show corresponding $95 \% \mathrm{Cls}$. At a GCS-P of 15 these $\mathrm{Cls}$ are wide, because that data point is underrepresented in the data set as a result of the inclusion criteria of the CRASH and IMPACT studies (see Table 3). Figure is available in color online only. 


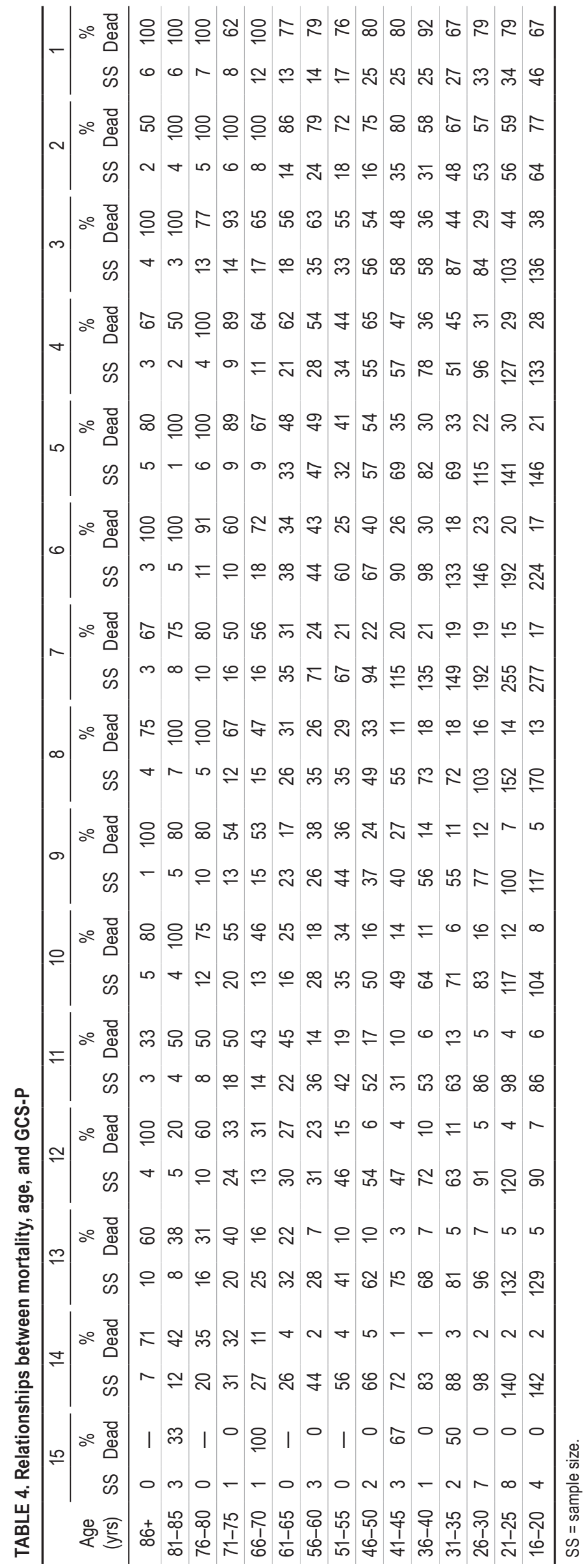

G. D. Murray et al. 

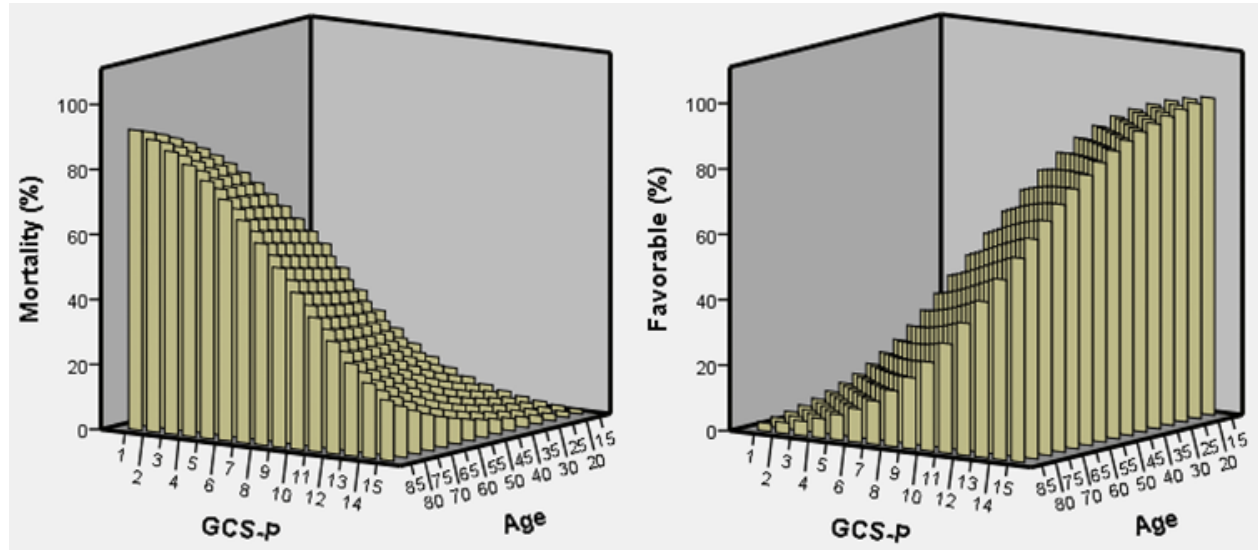

FIG. 3. Relationships between mortality, age, and GCS-P (left) and between favorable outcome, age, and GCS-P (right), based on the fitted logistic regression models. Figure is available in color online only.

combine the data. Values for the GCS score, pupil reactivity, and age, alone or in combination, are shown in Table 6. The combination of GCS-P and age was found to be substantially more informative than the GCS-P, age, GCS score, or pupil reactivity alone. The 3D plots in Fig. 3 illustrate the general nature of the relationships. To translate these graphs into a useable prognostic aid that provides precise percentage probabilities of outcomes with specific combinations of age and GCS-P, we produced the 2D charts shown in Fig. 4: GCS-P plus age (GCS-PA) prediction charts.

In these charts, the axes for age and GCS-P each start with the value associated with the worst prognosisGCS-P of 1 and age of 85 years-so that combinations toward the bottom left of the charts represent an increasing risk of mortality (Fig. 4 left) and a reduced prospect of favorable outcome (Fig. 4 right). Conversely, movement toward the combinations shown at the top right of the charts reflects a decreasing likelihood of mortality (Fig. 4 left) and an increasing likelihood of favorable outcome (Fig. 4 right). Color shading was used to highlight gradations in the relevant predicted probabilities as they range from $0 \%$ to $100 \%$.

The figures given for age represent precise values, as the probabilities in the charts are derived from the fitted logistic regression models. So, for example, if applying the chart for an individual 42 years of age, the relevant probability will lie between those probabilities given for 40 and 45 years.

\section{CT Characteristics and Outcome}

Given the $3 \mathrm{CT}$ abnormalities abstracted from the database (intracranial hematoma, absent cisterns, and subarachnoid hemorrhage [SAH]), there are 8 possible patterns of CT abnormality (e.g., hematoma alone, or no hematoma but absent cisterns and SAH). Table 7 shows the relationship between outcome and the presence of each of the 3 CT abnormalities, both individually and in all possible combinations. In addition, the corresponding information is presented for a simple grouping based on the number of CT abnormalities $(0,1$, or $\geq 2)$.

\section{Interactions Between CT Characteristics, GCS-P, Age, and Outcome}

Taken individually, differences in outcome for those patients with and without each abnormality are very similar for the 3 CT features. Taken in combination there is a gradation in risk moving from no CT abnormality (10\% mortality) to 1 abnormality (mortality $20 \%-28 \%$ ) to 2 abnormalities (mortality 38\%-53\%), and last to all $3 \mathrm{ab}-$ normalities (mortality 61\%). Fewer than 5\% of all patients (505/10,702 patients) fell into the highest risk category, that is, with all $3 \mathrm{CT}$ abnormalities present.

Table 8 shows the predictive yield of the CT data in terms of Nagelkerke's $\mathrm{R}^{2}$. The CT data are clearly informative, both in isolation and in their added value over age and GCS-P. There is a trade-off between predictive yield and simplicity as the number of CT groupings reduces from 8 to 4 and then to 3 ; although even with only 3 groups, most of the predictive yield is maintained. This leads to a simple extension of the earlier charts by stratifying the original charts by the $3 \mathrm{CT}$ groupings: no CT abnormality; exactly $1 \mathrm{CT}$ abnormality; and 2 or more $\mathrm{CT}$ abnormalities. The resulting GCS-PA CT prediction charts are shown in Figs. 5 and 6 . These charts were derived by adding the 3 -group CT variable as a categorical variable into the logistic regression model that includes age and GCS-P as linear terms.

TABLE 6. Predictive yield of GCS-P score and patient age-both separately and in combination

\begin{tabular}{lcc}
\hline \multirow{2}{*}{ Model } & \multicolumn{2}{c}{ Nagelkerke's R R $\left.^{2} \%\right)$} \\
\cline { 2 - 3 } & $\begin{array}{c}\text { Modeling } \\
\text { Death }\end{array}$ & $\begin{array}{c}\text { Modeling Favorable } \\
\text { Outcome }\end{array}$ \\
\hline GCS score as linear variable & 15.5 & 19.8 \\
\hline Pupil reactivity as linear variable & 14.0 & 13.9 \\
\hline GCS-P as linear variable & 18.4 & 22.2 \\
\hline Age as linear variable & 7.1 & 7.6 \\
\hline $\begin{array}{c}\text { GCS-P as linear variable \& age } \\
\text { as linear variable }\end{array}$ & 26.7 & 31.5 \\
\hline
\end{tabular}



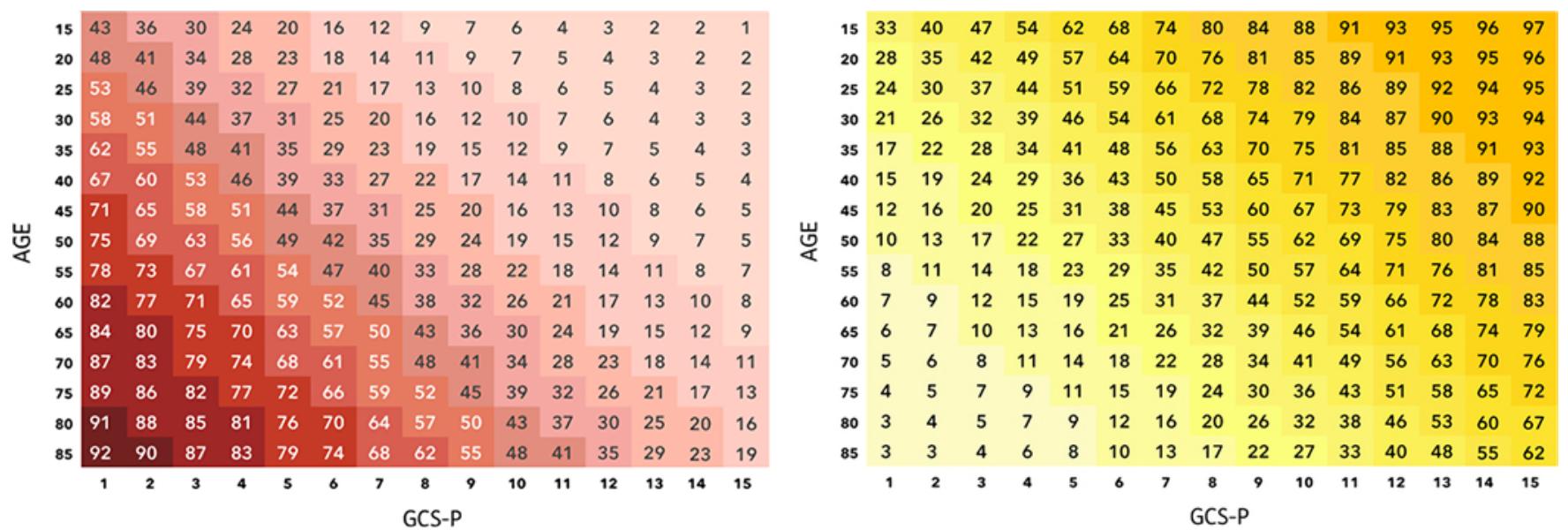

FIG. 4. GCS-PA prediction charts for the probability of 6-month mortality (left) and favorable outcome (right) based on the patient's admission GCS-P (derived as the GCS total score minus the number of nonreactive pupils) and age. The probabilities are derived from the fitted logistic regression models. Figure is available in color online only.

In comparison with published predictive models ${ }^{38,49}(\mathrm{Ta}-$ ble 8) our CT-stratified charts are superior to the IMPACT Core Model and the CRASH Basic Model. For patients included in the IMPACT database, the performance lies midway between the IMPACT Core and Extended models; and for patients in the CRASH study, the performance of the charts lies very close to that of the relatively complex CRASH CT Model.

\section{Discussion}

We have devised a method for bringing together the 4 most important prognostic features for outcome following head injury (early GCS score, pupil response, patient age, and CT findings) to display graphically their combined effects on the risk of death or prospects for independent recovery.

Much of the focus on outcome prediction in neurotrauma and, indeed, in medicine in general, has been on the methodology of prediction rather than on the method by which prognostic information is communicated, with even less attention paid to its practical impact. Robust tools for communicating risk in a readily understandable manner to doctors, other health care professionals, patients, and caregivers are needed to address the gap between the availability of prognostic evidence and its routine use in clinical practice-the so-called "prognostic gap." 32 There is a conceptual difficulty in applying probabilistic statistics (for example, $72 \%$ of patients with this condition survive) to the binary outcomes possible for an individual patient (survival or death). This contributes to the observation that clinicians systematically overlook relevant factors with prognostic significance in their decision making, leading to variations in predictions about patient outcomes and a tendency to pessimism. ${ }^{32}$

The process that we have developed for determining outcome probability in patients with TBI is easy and quick. Other than subtraction of pupil findings from the GCS score and identification of whether there are 0,1 , or 2 or more types of CT abnormality, our method does not require any calculation or computation. The distribution of probabilities from our charts can be applied to individual patients to inform and assist discussions about management both between clinicians and among clinicians, patients, and patients' relatives. The probabilities place outcome for an individual in the broader context of the influence and interactions of age and clinical and CT findings. The probability charts do not attempt to provide information on what will happen to a specific patient; that is more a prophesy of specific outcome and is recognized by clinicians as inherently fallible.

Multivariate prognostic models in head injury have not found widespread adoption in clinical practice for many reasons. The inherent uncertainty of models, set against the discipline and additional work involved in collecting and inputting multiple data points, contributes to the lack of adoption. ${ }^{20,30,35,39}$ A systematic review of prognostic models in TBI observed that only $10 \%$ of models were reported in a manner practical in the clinical setting. ${ }^{39}$ Clinicians need to understand the origin of information that they use to justify their practice. ${ }^{3}$ For clinical utility a model needs to balance incorporation of key data points and simplicity of use. ${ }^{54}$ For prognostic models to be useful, they should be presented in a user-friendly manner so they can be easily applied in the clinical scenario. ${ }^{38}$ The GCS-PA CT charts, which we present in this paper, achieve this. Our charts incorporate 4 factors-patient age, GCS score, pupil reactivity, and CT appearance-that have been extensively validated in previous studies as the most important prognostic features in head-injured patients..$^{28,38,49} \mathrm{We}$ focused on adults 16 years or older. Patient recruitment to the IMPACT and CRASH databases, on which our analysis was performed, was largely restricted to adults; even in the combined data, only 334 patients were children, compared with the 10,702 adults used for prognostic modeling in this paper. Age was treated as a linear factor on the logistic scale, as it was previously demonstrated that the association between age and both mortality and unfavorable outcome in neurotrauma is adequately described in this manner. ${ }^{17}$ 
TABLE 7. Relationships between CT characteristics and proportions of patients who either were dead or had favorable outcomes at 6 months after head injury

\begin{tabular}{|c|c|c|c|}
\hline CT Characteristic & Sample Size & Mortality & Favorable Outcome \\
\hline \multicolumn{4}{|l|}{ Hematoma } \\
\hline No & 6705 & $1223(18.2)$ & $4389(65.5)$ \\
\hline Yes & 3997 & $1455(36.4)$ & $1868(46.7)$ \\
\hline \multicolumn{4}{|l|}{ Absent cisterns } \\
\hline No & 8051 & 1551 (19.3) & $5184(64.4)$ \\
\hline Yes & 2651 & $1127(42.5)$ & $1073(40.5)$ \\
\hline \multicolumn{4}{|l|}{$\mathrm{SAH}$} \\
\hline No & 6765 & $1294(19.1)$ & $4451(65.8)$ \\
\hline Yes & 3937 & $1384(35.2)$ & $1806(45.9)$ \\
\hline No abnormality & 3588 & $350(9.8)$ & $2763(77.0)$ \\
\hline Hematoma alone & 1893 & $469(24.8)$ & $1107(58.5)$ \\
\hline $\begin{array}{l}\text { Absent cisterns } \\
\text { alone }\end{array}$ & 843 & $240(28.5)$ & $429(50.9)$ \\
\hline SAH alone & 1412 & $287(20.3)$ & $838(59.3)$ \\
\hline $\begin{array}{l}\text { Hematoma \& absent } \\
\text { cisterns }\end{array}$ & 441 & $235(53.3)$ & $152(34.5)$ \\
\hline Hematoma \& SAH & 1158 & $445(38.4)$ & $476(41.1)$ \\
\hline $\begin{array}{l}\text { Absent cisterns \& } \\
\text { SAH }\end{array}$ & 862 & $346(40.1)$ & $359(41.6)$ \\
\hline $\begin{array}{l}\text { Hematoma \& absent } \\
\text { cisterns \& SAH }\end{array}$ & 505 & $306(60.6)$ & $133(26.3)$ \\
\hline \multicolumn{4}{|l|}{$\begin{array}{l}\text { No. of CT abnor- } \\
\text { malities }\end{array}$} \\
\hline 0 & 3588 & $350(9.8)$ & $2763(77.0)$ \\
\hline 1 & 4148 & $996(24.0)$ & $2374(57.2)$ \\
\hline 2 or more & 2966 & $1332(44.9)$ & $1120(37.8)$ \\
\hline
\end{tabular}

Values represent number of patients (\%).

Attempts have been made to simplify the assessment of impaired consciousness by taking into account only 1 or 2 components of the Glasgow Coma Scale, contracting the criteria within these, or adding other features. ${ }^{15,47,52} \mathrm{Al}-$ though in severe head injury, defined as a GCS score of 8 or less, it is inevitable that changes in the motor score constitute the main influence on prognosis, a recent definitive analysis of 54,069 patients with TBI confirmed that each of the 3 components of the Glasgow Coma Scale contributes usefully to prognosis across the spectrum of the GCS total score. ${ }^{42}$

A range of CT findings have been shown individually to relate to prognosis in head injury. ${ }^{29,41,42,51}$ These have been combined in a variety of ways in different approaches to classification and scoring, and there is no consensus about a uniform system..$^{21,26}$ We were clear that for practical impact our approach needed to have clinical face value, to use a very limited number of categories to produce charts with distinctly different patterns, and to be as simple and easy to apply as possible. The finding that the presence of an intracranial hematoma, an abnormality of the basal cisterns, or SAH on CT scans had very comparable effects on prognosis supports our approach based on the number of these abnormalities present rather than a complex matrix of findings for each. In this way, we have been able
TABLE 8. Predictive yield of CT scan features alone and in combination with GCS-P and patient age, together with a comparison with the published IMPACT ${ }^{49}$ and $\mathrm{CRASH}^{38}$ predictive models

\begin{tabular}{|c|c|c|}
\hline \multirow[b]{2}{*}{ Model } & \multicolumn{2}{|c|}{ Nagelkerke's $\mathrm{R}^{2}(\%)$} \\
\hline & $\begin{array}{l}\text { Modeling } \\
\text { Death }\end{array}$ & $\begin{array}{l}\text { Modeling } \\
\text { Favorable } \\
\text { Outcome }\end{array}$ \\
\hline $\begin{array}{l}\text { CT scan showing every combination of abnor- } \\
\text { malities (8 groups) }\end{array}$ & 15.8 & 13.4 \\
\hline CT scan showing $0-3$ abnormalities (4 groups) & 15.1 & 13.1 \\
\hline $\begin{array}{l}\text { CT scan showing } 0,1 \text {, or } \geq 2 \text { abnormalities ( } 3 \\
\text { groups) }\end{array}$ & 14.4 & 12.7 \\
\hline $\begin{array}{l}\text { GCS-P as linear variable \& age as linear } \\
\text { variable }\end{array}$ & 26.7 & 31.5 \\
\hline $\begin{array}{l}\text { GCS-P as linear variable \& age as linear vari- } \\
\text { able \& CT findings in } 8 \text { groups }\end{array}$ & 33.5 & 35.5 \\
\hline $\begin{array}{l}\text { GCS-P as linear variable \& age as linear vari- } \\
\text { able \& CT findings in } 4 \text { groups }\end{array}$ & 32.6 & 35.2 \\
\hline $\begin{array}{l}\text { GCS-P as linear variable \& age as linear vari- } \\
\text { able \& CT findings in } 3 \text { groups }\end{array}$ & 32.1 & 34.9 \\
\hline \multicolumn{3}{|l|}{ IMPACT patients } \\
\hline IMPACT Core Model & 20.3 & 24.9 \\
\hline IMPACT Extended Model & 27.0 & 32.5 \\
\hline Age/GCS-P/CT chart & 23.9 & 28.8 \\
\hline \multicolumn{3}{|l|}{ CRASH patients } \\
\hline CRASH Basic Model & 34.9 & 37.7 \\
\hline CRASH CT Model & 41.9 & 42.1 \\
\hline Age/GCS-P/CT chart & 39.7 & 39.7 \\
\hline
\end{tabular}

to divide patients based on their CT findings into 3 approximately comparable size groups, with a clear gradation in prognosis, based on a simple count of the number of abnormalities. In patients with a mass lesion, prognosis is better when an extradural hematoma is present; however, this distinction was not made in the CRASH study and we draw attention to the need to allow for this. ${ }^{16,24,33,43}$ There is no evidence that other imaging features have a strong enough independent effect to merit additional categories. Using our present approach, the CT findings clearly add value to the predictive yield from age, GCS score, and pupil reactivity when assessed using Nagelkerke's $\mathrm{R}^{2}$.

The IMPACT and CRASH data are now more than a decade old, but comparisons within the IMPACT data set do not indicate a time-related influence. ${ }^{33}$ Moreover, despite changing practices in prehospital care, emergency medicine, and intensive care, neither a clear decrease in TBI-related mortality nor an improvement in overall outcome has been observed over the past 2 decades. ${ }^{44}$ Currently, several large prospective studies are underway, including in North America TRACK-TBI (Transforming Research and Clinical Knowledge in Traumatic Brain Injury, https://tracktbi.ucsf.edu//) $)^{54}$ and the TBI-Prognosis Study (http://www.tbi-prognosis.ca/) and in Europe CENTER-TBI (Collaborative European NeuroTrauma Effectiveness Research in TBI, https://www.center-tbi.eu//. ${ }^{25}$ 


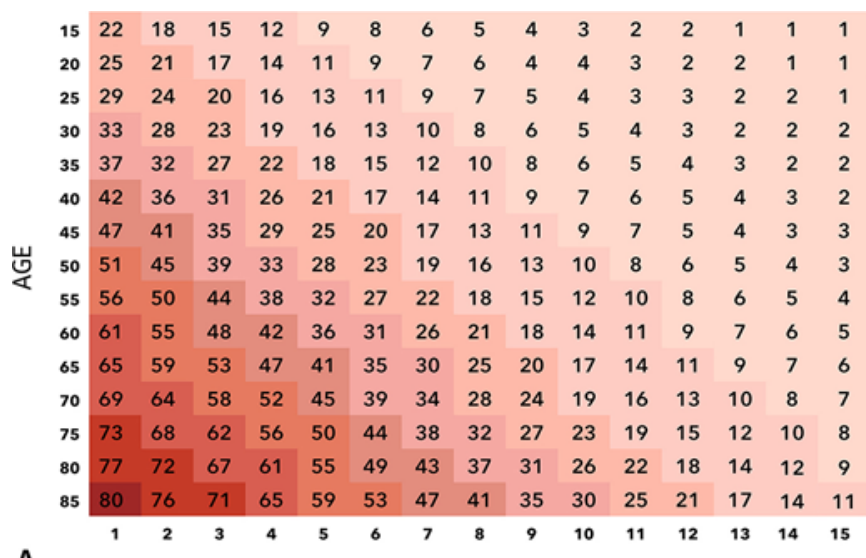

A

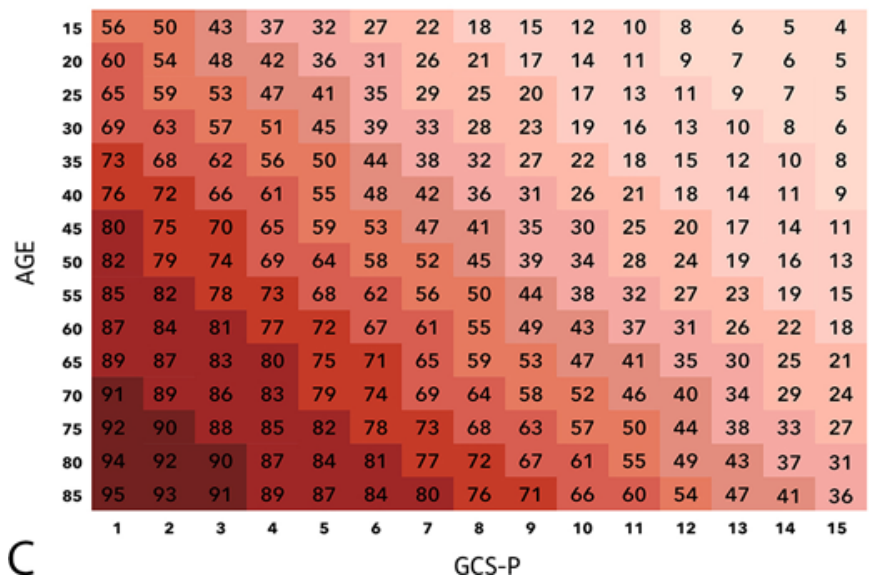

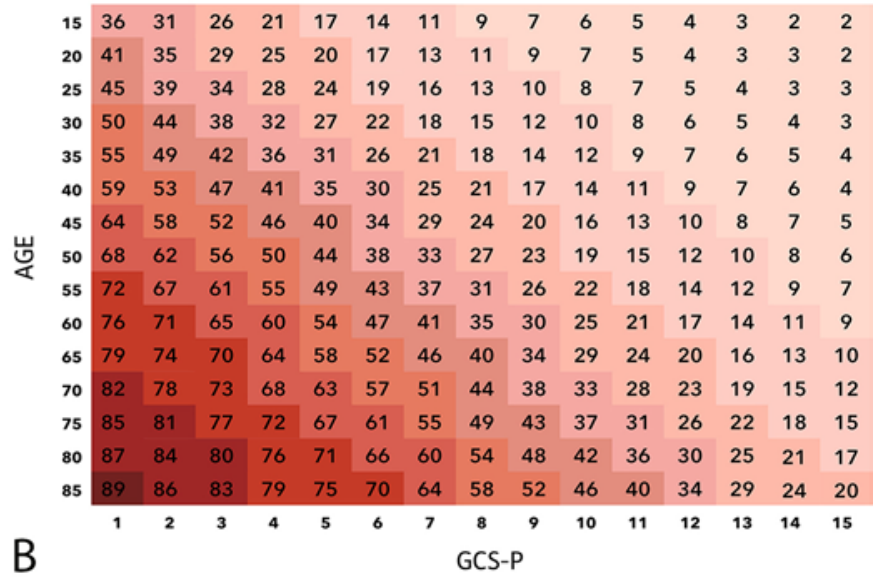

FIG. 5. GCS-PA CT prediction charts for the probability of mortality 6 months after head injury based on the patient's admission GCS-P (derived as the GCS sum score minus the number of nonreactive pupils) and age with no CT abnormality (A), exactly 1 CT abnormality (B), and 2 or more CT abnormalities (C). The category CT abnormalities comprises intracranial hematoma, absent cisterns, and $\mathrm{SAH}$. Figure is available in color online only.

These studies are linked together by the International Initiative for Traumatic Brain Injury Research (InTBIR; https://intbir.nih.gov//27 and are expected to yield detailed, standardized information based on almost 10,000 patients. These data will provide new and consistent information with which our modeling can be updated. ${ }^{31}$

Any prognostic model is by definition a simplification of reality, and in terms of Nagelkerke's $\mathrm{R}^{2}$ the models that we have derived using age, GCS, pupils, and CT findings explain approximately one-third of the observed variability in mortality and favorable outcome. The gain from using a more complex multivariable analysis such as the IMPACT and CRASH models is modest. ${ }^{49} \mathrm{~A}$ much repeated aphorism, ascribed to the statistician George Box, states that all models are wrong, but some are useful. We have strived to base our models on rigorous statistical principles, while placing a major emphasis on transparency and clinical face validity, to derive a decision aid that should be widely applicable and useful in clinical practice.

There is a strong theme running throughout the literature on clinical predictive models that simple models perform almost as well as complex ones. ${ }^{22,49}$ The performance of our CT-stratified charts lay midway between the performance of the Core and Extended IMPACT models and very close to that of the relatively complex CRASH CT Model. We believe this shows that our charts strike a good compromise in terms of performance versus simplicity.

Experience in risk estimation tools in other domains of medicine has underlined the fact that the outcomes identified need to be relevant to management decisions. ${ }^{23}$ There are continuing concerns about quality of survival after severe injury. Thus the DECRA (Decompressive Craniectomy) ${ }^{10}$ and RESCUEicp (Randomised Evaluation of Surgery with Craniectomy for Uncontrollable Elevation of Intracranial Pressure) ${ }^{18}$ trials demonstrated how surgical intervention with decompressive craniectomy in headinjured patients can increase survival, but at the expense of an increased proportion of patients left with poor functional status. The mortality and recovery outcomes that we have used are therefore of critical importance in discussions about the management of patients with neurotrauma.

Setting out the steps in arriving at a prediction has been recommended as a way to gain clinician acceptance..$^{53} \mathrm{~A}$ visual representation of the steps involved in making a prediction in head injury was first reported by Barlow et 


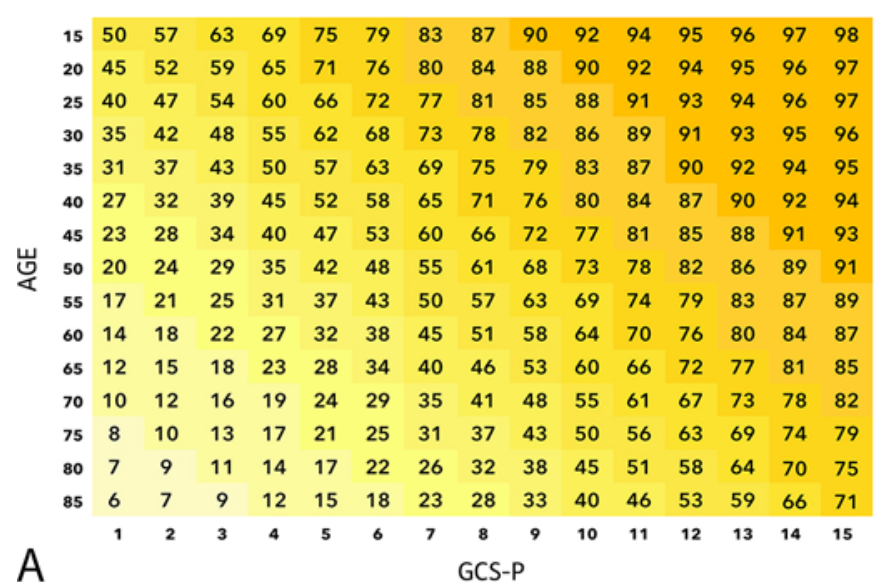

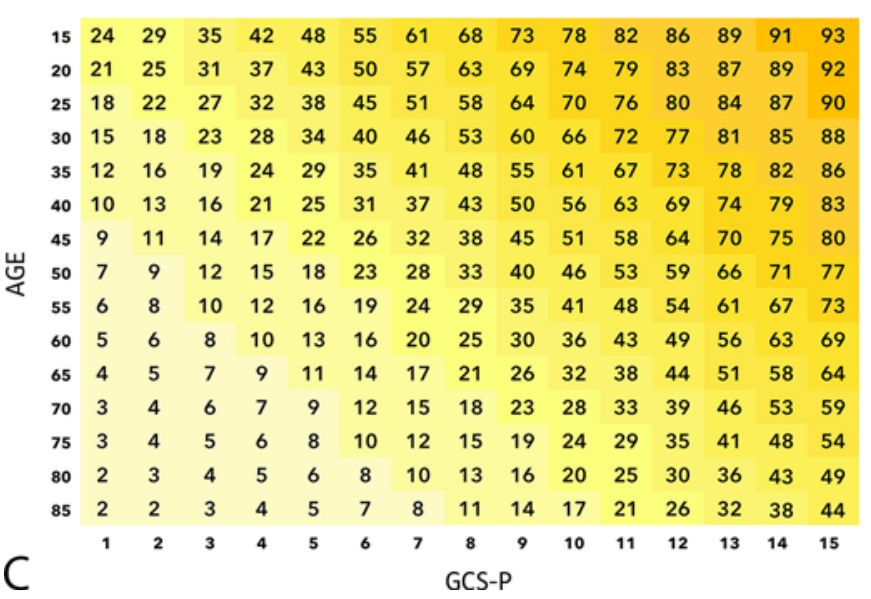

FIG. 6. GCS-PA CT prediction charts for the probability of favorable outcome 6 months after head injury based on the patient's admission GCS-P (derived as the GCS sum score minus the number of nonreactive pupils) and age with no CT abnormality (A), exactly 1 CT abnormality (B), and 2 or more CT abnormalities (C). The category CT abnormalities comprises intracranial hematoma, absent cisterns, and SAH. Figure is available in color online only.

al. ${ }^{11}$ The effects of successive incorporation of 8 predictive features in a Bayesian analysis on the changing relative likelihoods of death, of severely disabled survival, or of moderate good recovery was illustrated by changing positions of a point in a triangle. A prospective, clusterrandomized research study demonstrated that provision of this information was associated with changes in management, but the system was not taken up in routine practice. ${ }^{34}$

Decision trees, also termed classification and regression trees (CARTs), also have been proposed as a way to follow the steps in deriving a prediction of the single most likely outcome for a head-injured person. There are limitations in these approaches that may account for their lack of adoption. The decision tree method assumes the existence of break points in relationships between prognostic factors. This is not the case with these prognostic factors. In contrast, our approach is consistent with well-established continuous relationships between both age and GCS score and outcomes. Another problem is that the data sets used to derive the trees in an earlier study were very small, containing only $345-555$ patients, so that the artificial break points are likely to be inconsistent, reducing the validity and general applicability of the models. ${ }^{8,37,45,50}$ Although the tree identifies the single most likely outcome for an individual, the actual probability is not easily determined.

We are aware of only 1 previous example of charts of probabilities derived from a logistic regression model that was used to provide information about prognosis after a head injury. ${ }^{9}$ Choi et al. studied ventilated, severely head injured patients, and their model incorporated GCS score, oculocephalic responses, and age. ${ }^{9}$ Four different charts were provided, depending on the day after head injury and the oculocepahlic reflex result. The chart was not taken up generally, and Choi and colleagues later moved on to a decision tree method. ${ }^{8}$ Factors in this lack of success may have been restriction of the model to patients in the intensive care unit, the requirement for assessment of the oculocepahlic reflex, and the yield of information about only the probability of good recovery and not mortality. Furthermore, the charts were composed of a series of boundary lines based on deciles of outcome rather than on a given GCS score or age, and how these were to be applied to an individual patient was not readily appreciated.

Our work differs from that of Choi and colleagues in that we incorporated pupil reactivity as a simpler and more widely assessed feature than the oculocephalic reflex, and 
we added extra information from the CT scan. Also, our charts cover patients at all levels of head injury severity and predict mortality as well as good functional outcome. They thus address a greater scope of neurotrauma in a more relevant and more informative, understandable way.

Risk prediction charts have been produced in other fields of medicine. Our approach derives from possibly the best-known uses of these, identification of the risks of cardiovascular disease. In North America, data were derived from the Framingham Study to create a risk score calculator based on factors including blood pressure, total cholesterol, HDL-C, smoking, and diabetes..$^{13}$ How this interacts with age is shown in charts of absolute and relative risks with differing (arbitrary) degrees of risk distinguished by color coding. ${ }^{23}$ In Europe, the European Systematic Coronary Risk Evaluation (SCORE) algorithm ${ }^{40}$ charts evaluate the 10-year risk of fatal cardiovascular disease based on smoking, age, sex, systolic blood pressure, and total cholesterol. This results in a matrix of 16 charts in which risk progressively increases from female nonsmokers, age 40 years, with low blood pressure to the maximum risk in male smokers, age 65 years, with high blood pressure; the risks are illustrated with a transition from color overlays of green to yellow, orange, and red.

Our charts permit a rapid assessment of prognosis for neurotrauma patients. Color shading was used to highlight gradations in relevant predicted probabilities as they range from $0 \%$ up to $100 \%$. In real life, the GCS-P is quickly determined by subtracting the number of nonreactive pupils (the PRS) from the total GCS score. By reference to the GCS-PA chart, the GCS-P is read against the patient's age and an estimate of prognosis is determined. The simplicity of the GCS-PA model makes it ideal for application in acute clinical settings, where it can provide rapid stratification of patients as part of the information that guides discussions about clinical decisions. The charts can also support informed communication about patients with relatives and other care givers. The charts are also not intended to eliminate the use of formal multivariate modeling, which can be added to incorporate more complex information. The charts are decision-making tools that complement but can never replace clinical judgment. They will nevertheless reduce risk of influence from subjective, inappropriate assumptions. This is important because clinicians have been shown to systematically overestimate the likelihood of a poor outcome. ${ }^{32}$

Our aim in this work is to promote the use of predictive models in TBI by making predictions readily accessible in the clinical setting. The present paper establishes the principle that this can be achieved by a graphical display of information. Future work to update the charts with information from current and new studies will ensure that the tool remains useful. If new, powerful, and independent prognostic features are identified (for example, genetic factors or biomarkers) a new generation of charts can be produced, differentiated by the presence or absence of these features. Access to larger databases including pediatric patients with head injury will enable the principle to be applied to develop charts for children. Likewise, more data on mildly head injured patients, in whom there may be additional, different prognostic factors, will expand the value of the approach in this, the largest group of head-injured patients. ${ }^{46}$

\section{Conclusions}

The important prognostic features in neurotrauma can be brought together to display graphically their combined effects on the risks of death or prospects for independent recovery. The approach can support decision making and improve communication of risk among health care professionals, patients, and their relatives. These charts will not replace clinical judgment, but they will reduce the risk of influence from subjective, inappropriate assumptions.

\section{Acknowledgments}

We thank Professor Andrew Maas, University of Antwerp, and Professor Ian Roberts, London School of Hygiene and Tropical Medicine, for allowing the use of information from the IMPACT and CRASH databases, respectively. We are grateful to Luke Moodley of The Edinburgh Film Company for his assistance in developing the design of the prognostic charts.

\section{References}

1. Barlow P, Teasdale G: Prediction of outcome and the management of severe head injuries: the attitudes of neurosurgeons. Neurosurgery 19:989-991, 1986

2. Braakman R, Gelpke GJ, Habbema JD, Maas AI, Minderhoud JM: Systematic selection of prognostic features in patients with severe head injury. Neurosurgery 6:362-370, 1980

3. Brahams D, Wyatt J: Decision aids and the law. Lancet 2:632-634, 1989

4. Brennan PM, Murray GD, Teasdale GM: Simplifying the use of prognostic information in traumatic brain injury. Part 1: The GCS-Pupils score: an extended index of clinical severity. J Neurosurg [epub ahead of print April 10, 2018. DOI: 10.3171/2017.12.JNS172780]

5. Brust-Renck PG, Royer CE, Reyna VF: Communicating numerical risk: human factors that aid understanding in health care. Rev Hum Factors Ergon 8:235-276, 2013

6. Ceriani E, Combescure C, Le Gal G, Nendaz M, Perneger $\mathrm{T}$, Bounameaux $\mathrm{H}$, et al: Clinical prediction rules for pulmonary embolism: a systematic review and meta-analysis. J Thromb Haemost 8:957-970, 2010

7. Chesnut RM, Ghajar J, Maas AIR: Guidelines for the management and prognosis of severe traumatic brain injury. Part 2: Early indicators of prognosis in severe traumatic brain injury. J Neurotrauma 17:555-627, 2000

8. Choi SC, Muizelaar JP, Barnes TY, Marmarou A, Brooks DM, Young HF: Prediction tree for severely head-injured patients. J Neurosurg 75:251-255, 1991

9. Choi SC, Ward JD, Becker DP: Chart for outcome prediction in severe head injury. J Neurosurg 59:294-297, 1983

10. Cooper DJ, Rosenfeld JV, Murray L, Arabi YM, Davies AR, D'Urso P, et al: Decompressive craniectomy in diffuse traumatic brain injury. N Engl J Med 364:1493-1502, 2011

11. Corbett W (ed): Medical Applications of Microcomputers. New York: Wiley, 1987

12. Counsell C, Dennis M: Systematic review of prognostic models in patients with acute stroke. Cerebrovasc Dis 12:159170,2001

13. D’Agostino RB Sr, Vasan RS, Pencina MJ, Wolf PA, Cobain M, Massaro JM, et al: General cardiovascular risk profile for use in primary care: the Framingham Heart Study. Circulation 117:743-753, 2008

14. Garcia-Retamero R, Cokely ET, Hoffrage U: Visual aids 
improve diagnostic inferences and metacognitive judgment calibration. Front Psychol 6:932, 2015

15. Gill M, Windemuth R, Steele R, Green SM: A comparison of the Glasgow Coma Scale score to simplified alternative scores for the prediction of traumatic brain injury outcomes. Ann Emerg Med 45:37-42, 2005

16. Haselsberger K, Pucher R, Auer LM: Prognosis after acute subdural or epidural haemorrhage. Acta Neurochir (Wien) 90:111-116, 1988

17. Hukkelhoven CWPM, Steyerberg EW, Rampen AJJ, Farace E, Habbema JDF, Marshall LF, et al: Patient age and outcome following severe traumatic brain injury: an analysis of 5600 patients. J Neurosurg 99:666-673, 2003

18. Hutchinson PJ, Kolias AG, Timofeev IS, Corteen EA, Czosnyka M, Timothy J, et al: Trial of decompressive craniectomy for traumatic intracranial hypertension. N Engl J Med 375:1119-1130, 2016

19. Kansagara D, Englander H, Salanitro A, Kagen D, Theobald C, Freeman M, et al: Risk prediction models for hospital readmission: a systematic review. JAMA 306:1688-1698, 2011

20. Latronico N: Prediction is very difficult, especially about the future. Crit Care Med 43:505-506, 2015

21. Marshall LF, Marshall SB, Klauber MR, Marjan van Berkum C, Eisenberg HM, Jane JA, et al: A new classification of head injury based on computerized tomography. J Neurosurg 75 (1 Suppl):S14-S20, 1991

22. Lingsma HF, Roozenbeek B, Steyerberg EW, Murray GD, Maas AI: Early prognosis in traumatic brain injury: from prophecies to predictions. Lancet Neurol 9:543-554, 2010

23. Lloyd-Jones DM: Cardiovascular risk prediction: basic concepts, current status, and future directions. Circulation 121:1768-1777, 2010

24. Maas AIR, Hukkelhoven CWPM, Marshall LF, Steyerberg EW: Prediction of outcome in traumatic brain injury with computed tomographic characteristics: a comparison between the computed tomographic classification and combinations of computed tomographic predictors. Neurosurgery 57:1173-1182, 2005

25. Maas AIR, Menon DK, Steyerberg EW, Citerio G, Lecky F, Manley GT, et al: Collaborative European NeuroTrauma Effectiveness Research in Traumatic Brain Injury (CENTERTBI): a prospective longitudinal observational study. Neurosurgery 76:67-80, 2015

26. Maas AIR, Steyerberg EW, Butcher I, Dammers R, Lu J, Marmarou A, et al: Prognostic value of computerized tomography scan characteristics in traumatic brain injury: results from the IMPACT study. J Neurotrauma 24:303-314, 2007

27. Manley GT, Maas AIR: Traumatic brain injury: an international knowledge-based approach. JAMA 310:473-474, 2013

28. Marmarou A, Lu J, Butcher I, McHugh GS, Mushkudiani NA, Murray GD, et al: IMPACT database of traumatic brain injury: design and description. J Neurotrauma 24:239-250, 2007

29. Marshall LF, Gautille T, Klauber MR, Eisenberg HM, Jane JA, Luerssen TG, et al: The outcome of severe closed head injury. J Neurosurg 75 (1 Suppl):S28-S36, 1991

30. Menon DK, Zahed C: Prediction of outcome in severe traumatic brain injury. Curr Opin Crit Care 15:437-441, 2009

31. Moons KGM, Kengne AP, Grobbee DE, Royston P, Vergouwe Y, Altman DG, et al: Risk prediction models: II. External validation, model updating, and impact assessment. Heart 98:691-698, 2012

32. Moore NA, Brennan PM, Baillie JK: Wide variation and systematic bias in expert clinicians' perceptions of prognosis following brain injury. Br J Neurosurg 27:340-343, 2013

33. Murray GD, Butcher I, McHugh GS, Lu J, Mushkudiani NA, Maas AIRR, et al: Multivariable prognostic analysis in traumatic brain injury: results from the IMPACT study. J Neurotrauma 24:329-337, 2007

34. Murray LS, Teasdale GM, Murray GD, Jennett B, Miller JD,
Pickard JD, et al: Does prediction of outcome alter patient management? Lancet 341:1487-1491, 1993

35. Mushkudiani NA, Hukkelhoven CWPM, Hernández AV, Murray GD, Choi SC, Maas AIR, et al: A systematic review finds methodological improvements necessary for prognostic models in determining traumatic brain injury outcomes. $\mathbf{J}$ Clin Epidemiol 61:331-343, 2008

36. Nagelkerke NJD: A note on a general definition of the coefficient of determination. Biometrika 78:691-692, 1991

37. Pang BC, Kuralmani V, Joshi R, Hongli Y, Lee KK, Ang BT, et al: Hybrid outcome prediction model for severe traumatic brain injury. J Neurotrauma 24:136-146, 2007

38. Perel P, Arango M, Clayton T, Edwards P, Komolafe E, Poccock S, et al: Predicting outcome after traumatic brain injury: practical prognostic models based on large cohort of international patients. BMJ 336:425-429, 2008

39. Perel P, Edwards P, Wentz R, Roberts I: Systematic review of prognostic models in traumatic brain injury. BMC Med Inform Decis Mak 6:38, 2006

40. Perk J, De Backer G, Gohlke H, Graham I, Reiner Z, Verschuren M, et al: European Guidelines on cardiovascular disease prevention in clinical practice (version 2012). The Fifth Joint Task Force of the European Society of Cardiology and Other Societies on Cardiovascular Disease Prevention in Clinical Practice (constituted by representatives of 9 societies and by invited experts). Eur Heart J 33:1635-1701, 2012

41. Raj R, Siironen J, Skrifvars MB, Hernesniemi J, Kivisaari R: Predicting outcome in traumatic brain injury: development of a novel computerized tomography classification system (Helsinki computerized tomography score). Neurosurgery 75:632-647, 2014

42. Reith FCM, Lingsma HF, Gabbe BJ, Lecky FE, Roberts I, Maas AIR: Differential effects of the Glasgow Coma Scale Score and its Components: an analysis of 54,069 patients with traumatic brain injury. Injury 48:1932-1943, 2017

43. Roberts I, Yates D, Sandercock P, Farrell B, Wasserberg J, Lomas G, et al: Effect of intravenous corticosteroids on death within 14 days in 10008 adults with clinically significant head injury (MRC CRASH trial): randomised placebo-controlled trial. Lancet 364:1321-1328, 2004

44. Roozenbeek B, Maas AIR, Menon DK: Changing patterns in the epidemiology of traumatic brain injury. Nat Rev Neurol 9:231-236, 2013

45. Rovlias A, Kotsou S: Classification and regression tree for prediction of outcome after severe head injury using simple clinical and laboratory variables. J Neurotrauma 21:886893,2004

46. Silverberg N, Gardner AJ, Brubacher J, Panenka W, Li JJ, Iverson GL: Systematic review of multivariable prognostic models for mild traumatic brain injury. J Neurotrauma 32:517-526, 2015

47. Starmark JE, Stålhammar D, Holmgren E: The Reaction Level Scale (RLS85). Manual and guidelines. Acta Neurochir (Wien) 91:12-20, 1988

48. Steyerberg EW, Moons KGM, van der Windt DA, Hayden JA, Perel P, Schroter S, et al: Prognosis Research Strategy (PROGRESS) 3: prognostic model research. PLoS Med 10:e1001381, 2013

49. Steyerberg EW, Mushkudiani N, Perel P, Butcher I, Lu J, McHugh GS, et al: Predicting outcome after traumatic brain injury: development and international validation of prognostic scores based on admission characteristics. PLoS Med 5:e165, 2008

50. Temkin NR, Holubkov R, Machamer JE, Winn HR, Dikmen SS: Classification and regression trees (CART) for prediction of function at 1 year following head trauma. J Neurosurg 82:764-771, 1995

51. Thelin EP, Nelson DW, Vehviläinen J, Nyström H, Kivisaari R, Siironen J, et al: Evaluation of novel computerized tomography 
scoring systems in human traumatic brain injury: An observational, multicenter study. PLoS Med 14:e1002368, 2017

52. Wijdicks EFM, Bamlet WR, Maramattom BV, Manno EM, McClelland RL: Validation of a new coma scale: the FOUR score. Ann Neurol 58:585-93, 2005

53. Wyatt JC, Altman DG: Commentary: Prognostic models: clinically useful or quickly forgotten? Br Med J 311:1539_ 1541, 1995

54. Yue JK, Vassar MJ, Lingsma HF, Cooper SR, Okonkwo DO, Valadka AB, et al: Transforming research and clinical knowledge in traumatic brain injury pilot: multicenter implementation of the common data elements for traumatic brain injury. J Neurotrauma 30:1831-1844, 2013

\section{Disclosures}

Financial support for this study was provided by the Muriel Cooke Bequest to the University of Glasgow.

\section{Author Contributions}

Conception and design: all authors. Acquisition of data: all authors. Analysis and interpretation of data: all authors. Drafting the article: all authors. Critically revising the article: all authors. Reviewed submitted version of manuscript: all authors. Approved the final version of the manuscript on behalf of all authors: Murray. Statistical analysis: all authors.

\section{Supplemental Information}

Copies of prognosis charts in Figs. 4, 5, and 6 are available to download online at www.glasgowcomascale.org.

\section{Companion Papers}

Brennan PM, Murray GD, Teasdale GM: Simplifying the use of prognostic information in traumatic brain injury. Part 1: The GCS-Pupils score: an extended index of clinical severity. DOI: 10.3171/2017.12.JNS172780.

\section{Correspondence}

Gordon Murray: Usher Institute of Population Health Sciences and Informatics, University of Edinburgh Medical School, Edinburgh, United Kingdom. gordon.murray@ed.ac.uk. 